Université de Strasbourg

Séminaire de Probabilités

$1974 / 75$

\title{
CORRECTIONS A DES EXPOSES DE 1973/74
}

par C. Dellacherie

D'abord deux petits amuse-gueule

1) Le théorème 2 de "Ensembles analytiques : ..." - p 338 - est inexact tel qu'il est écrit : il faut supposer de plus que l'ensemble $E_{n}$ appartient au pavage $E_{n}$ pour tout $n$, sauf éventuellement pour un nombre fini d'entiers.

2) Dans "Une propriété des ensembles semi-polaires" - p495 - , il faut lire, dans les premières lignes, "... changenert de temps par rapport à une fonctionnelle additive strictement croissante ET CONTINUE "

Passons au plat de résistance, consistant en "Une remarque sur les espaces sousliniens de Bourbaki" - p406 et 407 - . La meilleure correction consiste à prendre un pot de colle et à coller soigneusement la page 406 sur la page 407 ! En effet, la démonstration p407 est incomplète : on y caractérise seulement les fermés dont l'image par $f$ est un point, et il n'est pas vrai en général que l'ensemble E' considéré soit souslinien dans $\underset{F}{F}(P)$. Plus précisément, on peut montrer que $E^{\prime}$ est souslinien si et seulement si, pour tout ouvert U de $P$, $f(U)$ est borélien dans $E$. Cela n'implique pas par ailleurs que le théorème énoncé est faux : il reste simplement en l'état de conjecture. 